\title{
Adopting static typing in scientific projects
}

Predrag Gruevski \& Colin Carroll SciPy 2021 


\section{Speakers}

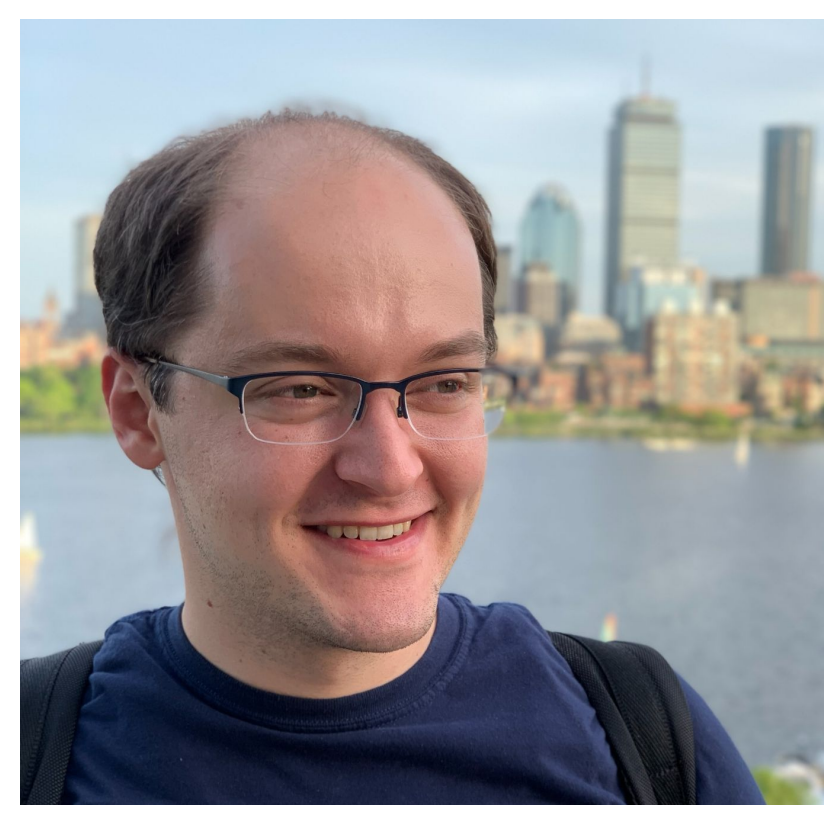

Predrag Gruevski

typing_copilot author

Twitter: @PredragGruevski

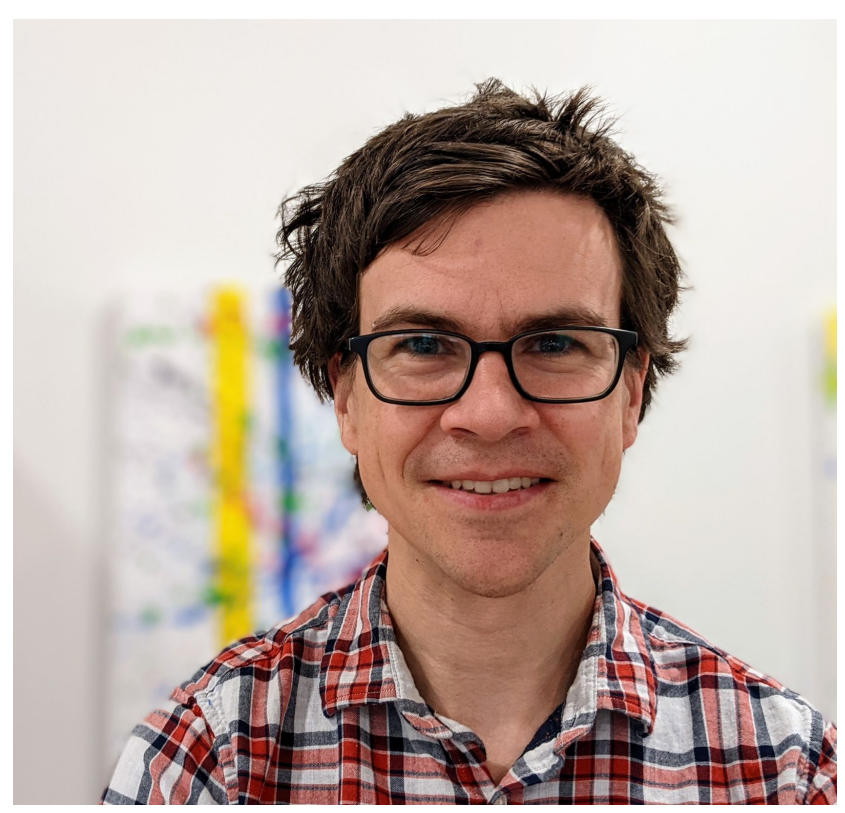

Colin Carroll

ArviZ maintainer

Twitter: @colindcarroll 


\section{Python static typing and type hints}

def square(a):

return a $\star a$

def square(a: int) $->$ int: return $a \star a$ 


\section{Outline}

- Difficulties when type-hinting a large existing codebase

- "Ratcheting" type-hinting workflow with typing_copilot

- Case studies on adopting typing_copilot 


\section{Outline}

- Difficulties when type-hinting a large existing codebase

- "Ratcheting" type-hinting workflow with typing_copilot

- Case studies on adopting typing_copilot 


\section{Three Stakeholder Personas}

E End-user Emily

Contributor Cathy

M Maintainer Michelle 


\section{Type-hinting a large existing codebase}

E End-user Emily

Wants new features!

- Type hints and features must be added concurrently

- Wants type hints exported to support her own codebase 


\section{Type-hinting a large existing codebase}

C Contributor Cathy

Might not be familiar w/ type-hinting. Might stop contributing if:

- Adding type hints is too difficult or too time-consuming

- Pull request reviews take too long 


\section{Type-hinting a large existing codebase}

M Maintainer Michelle

Needs to keep E and Chappy.

Also wants:

- Type hints used to prevent bugs

- Fully type-hinted new features

- Existing code allowed to be partially type-hinted

- Type hint coverage cannot degrade 


\section{Difficulties when adopting type-hinting}

Difficult up-front setup for M

- Different rules for different parts of the codebase

- Complex mypy config required

- Lots of config options, which are best?

=> Analysis paralysis! M might give up entirely... 


\section{Difficulties when adopting type-hinting}

Difficult up-front setup for $M$

- Different rules for different parts of the codebase

- Complex mypy config required

- Lots of config options, which are best?

=> Analysis paralysis! $M$ might give up entirely...

Ongoing difficulties for C and $M$

- Must ensure pull requests do not degrade type hint coverage

- Must update mypy config as type hint coverage improves

=> Two steps forward, one step back 


\section{Difficulties when adopting type-hinting}

Difficult up-front setup for $M$

- Different rules for different parts of the codebase

- Complex mypy config required

- Lots of config options, which are best?

=> Analysis paralysis! M might give up entirely...

Ongoing difficulties for $C$ and $M$

- Must ensure pull requests do not degrade type hint coverage

- Must update mypy config as type hint coverage improves

=> Two steps forward, one step back 


\section{Outline}

- Difficulties when type-hinting a large existing codebase

- "Ratcheting" type-hinting workflow with typing_copilot

- Case studies on adopting typing_copilot 


\section{typing_copilot}

Finds the strictest mypy config that passes with no errors

- Checks codebase with all mypy checks enabled

- Any checks that find errors are disabled for the affected files

- Everything else represents the strictest passing mypy config! 


\section{typing_copilot resolves type-hinting difficulties}

No analysis paralysis

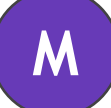

- The best mypy config is always the strictest passing one

- typing_copilot init 


\section{typing_copilot resolves type-hinting difficulties}

No analysis paralysis

- The best mypy config is always the strictest passing one

- typing_copilot init

Ratcheting workflow

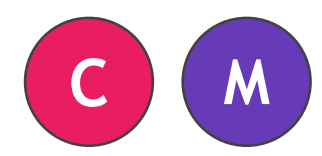

- Catch type correctness or type coverage regressions with mypy

- Then, try to generate a stricter passing mypy config and check it in

- On $\mathrm{Cl}$, ensure the mypy config is the strictest possible

- typing_copilot tighten [--error-if-can-tighten] 


\section{Outline}

- Difficulties when type-hinting a large existing codebase

- "Ratcheting" type-hinting workflow with typing_copilot

- Case studies on adopting typing_copilot 


\section{Actionable and minimal path to type-checking ArviZ}

Running mypy arviz produced 361 type errors!

- Most mypy checks are enabled by default

- Fixing hundreds of errors is not an encouraging starting point 


\section{Actionable and minimal path to type-checking ArviZ}

Running mypy arviz produced 361 type errors!

- Most mypy checks are enabled by default

- Fixing hundreds of errors is not an encouraging starting point

typing_copilot init produces the minimal set of errors

- Runs mypy with all optional checks disabled

- Any failures in that configuration must be fixed before proceeding

- ArviZ had 41 type errors with all optional checks disabled

- Multiple user-facing bugs fixed in the process, e.g.: https://github.com/arviz-devs/arviz/pull/1493 


\section{Sustainably improving type coverage in GraphQL compiler}

typing_copilot requires this module to be completely typed

Module foo (existing untyped code)

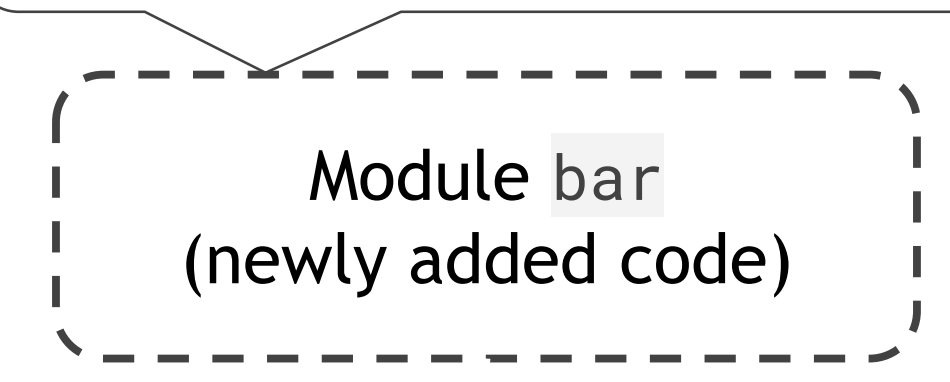




\section{Sustainably improving type coverage in GraphQL compiler}
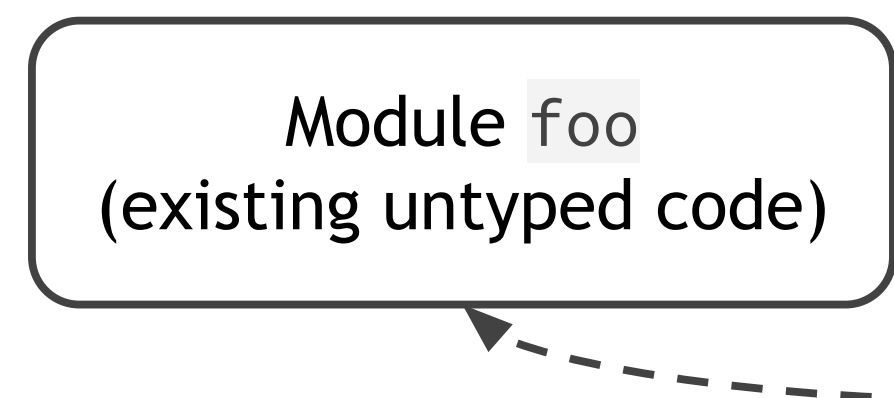

from foo import func

foo. func used in typed code, so must be type-hinted too

typing_copilot requires this module to be completely typed

Mypy's disallow_untyped_calls rule: "don't allow calling untyped functions from type-hinted code." 


\section{Bonus ratchet tightening on files you didn't modify}

$--$

$\vee \stackrel{+\uparrow}{*} 14 \square \square \square \square$ mypy.ini

This PR didn't modify the file referenced by this rule!

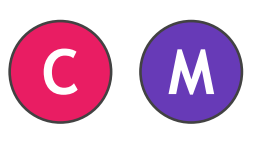

79 - [mypy-graphql_compiler.compiler.helpers.*]

80 - disallow_untyped_calls = False

Comment on lines -79 to -80

(19)

obi1kenobi on Aug 6, 2020 Author Member

This is perhaps my favorite part about typing-copilot — it catches and tightens settings I never would have thought about doing by hand. In this case, it noticed that graphql_compiler.compiler.helpers was calling a previously-untyped function that is now typed.

Since this suppression is no longer necessary, it is removed. That module is guaranteed to now and forever more only call fully-typed code.

https: / /github.com/kensho-technologies/graphql-compiler/pull/891/files\#r466729797 


\section{Try typing_copilot in your own project}

pip install typing_copilot

Repo: https://github.com/obi1kenobi/typing copilot

Examples of adopting typing_copilot + plugging into $\mathrm{Cl}$ :

https://github.com/kensho-technologies/graphql-compiler/pull/937

https://github.com/arviz-devs/arviz/pull/1528

https://github.com/arviz-devs/arviz/pull/1624

Talk to us on Twitter:

@PredragGruevski and @colindcarroll 